Background The average risk of infection after occupational exposure to HIV is $0.3 \%(0.2-0.5 \%$ percutaneous exposure, $0.01-0.5 \%$ contact with mucous or non-intact skin) and after sexual exposure $0.01-3 \%$, depending on sexual practise.

An action protocol has been in place at our centre since 2010, based on international recommendations for exposure to HIV that include:

1. Start treatment within 72 hours post-exposure

2. 1st choice guideline: Tenofovir/Emtricitabine+Lopinavir/ Ritonavir regimen or the source treatment if viral load is controlled; 2nd choice Tenofovir/Emtricitabine or Lamivudine/Zidovudine+ protease inhibitor(PI), boosted with Ritonavir.

3. Length of treatment: 30 days

4. Serological analysis at different points until the 6 th month.

Before 2010, the hospital followed the international recommendations, with 1st choice Tenofovir/Emtricitabine or Lamivudine/ Zidovudine + PI boosted with Ritonavir.

Purpose To evaluate the effectiveness and change to the protocol currently in force since 2010 and that of the previous international recommendations, following exposure to HIV.

Materials and Methods A retrospective observational study. Sample: $100 \%$ of patients with antiretroviral treatment following exposure. Period: January 2000-June 2012. Data Sources: Pharmacotherapy records (Silicon computer programme) and electronic medical records (IANUS application). Variable effectiveness: absence of seroconversion in exposed patient following post-exposure prophylactic treatment (PEPT). Analysis on: day- 0 , month- 1 , month- 3 and month-6.

Results 33 patients. Average age 37.3(23-65), 13 males (39.4\%). Patients treated with first choice: $94 \%$, other therapeutic options: $6.0 \%$. $90.9 \%$ of patients received treatment for 30 days. $38.2 \%$ of patients underwent correct serological monitoring until 6 months, $52.9 \%$ until 3 months. $96.9 \%$ started treatment within 72 hours of exposure. All baseline serologies were negative and there were no cases of seroconversion. Average cost/patient $€ 747$.

Conclusions PEPT was able to achieve the therapeutic goal in all study patients. The treatment chosen and the time of beginning after exposure were correct. The follow-up until 6 months was not carried out correctly in a significant percentage of patients. These facts and the high costs, require close pharmacotherapy monitoring of these patients.

No conflict of interest.

\section{CPC-044 EFFECTIVINESS AND SAFETY OF BEVACIZUMAB IN METASTATIC BREAST CANCER IN CLINICAL PRACTISE}

doi:10.1136/ejhpharm-2013-000276.501

'R Veiga, ' $G$ Durán, 'MJ Lamas, ${ }^{2} J F$ Cueva, 'B Bernárdez, ${ }^{2} \mathrm{~T}$ Curiel, 'E Espino, 'ME Concheiro. 'Complejo Hospitalario Universitario de Santiago de Compostela, Pharmacy, Santiago de Compostela, Spain; ${ }^{2}$ Complejo Hospitalario Universitario de Santiago de Compostela, Oncology, Santiago de Compostela, Spain

Background New data released by clinical trials AVADO and RIB$\mathrm{BON}$ have questioned the use of Avastin in metastatic breast cancer (MBC). EMA keeps the indication of first line in combination with paclitaxel or capecitabine when taxanes or anthracyclines are not indicated.

Purpose This study explores our single-centre experience to cheque the effectiveness and safety of bevacizumab in $\mathrm{MBC}$ in clinical practise.

Materials and Methods Retrospective study of $41 \mathrm{MBC}$ patients treated with bevacizumab and chemotherapy in a Spanish teaching hospital from $07 / 2008$ to $06 / 2012$. Toxic effects were graded according to the National Cancer Institute Common Toxicity Criteria (NCI-CTC). Disease status was assessed according to the Response
Evaluation Criteria in Solid Tumors (RECIST). Clinical evaluation included clinical response, time to progression (TTP), and toxicity. Median survival times were estimated from Kaplan-Meier curves. Data analysis was performed using SPSS-17.0.

Results Median age was 59 yrs (34-75). $87.8 \%$ of patients had ECOG PS 0-1. Bevacizumab was administered with docetaxe (46.3\%), paclitaxel (29.3\%), taxane-carboplatin (17.1\%) or capecitabine $(7.3 \%)$. It was used as first line in 19 cases $(46.3 \%)$, second line in 5 and following lines in 17 cases (41.5\%). Sites of metastases were: 26 visceral and 4 skeletal. Overall Response was 46.4\% (4.9\% Complete and $41.5 \%$ Partial). $17.1 \%$ had progressive disease. Median TTP: 7.8 months (6.5-9.2;95\%CI). Median TTP of first-line paclitaxel-bevacizumab was $11 \mathrm{vs.} 7.7$ months for the rest of the combinations $(\mathrm{P}=0.501)$. Safety outcomes were similar among treatments. G1-2 toxicities: bleeding (32\%), anaemia $(21.8 \%)$, mucositis (21.9\%), diarrhoea (9.7\%), hypertension (20\%). 1 patient suffered grade 4 hypertension resulting in discontinuation and 2 patients suffered deep vein thromboembolisms. Other non-specific toxicities: neutropenia $(31.2 \%-\mathrm{G} 3-4=$ $7.3 \%)$, neuropathy $(19.5 \%)$, alopecia $(24.4 \%)$, nausea/vomiting (9.8\%).

Conclusions TTP was longer with paclitaxel than with other antineoplastic agents but the difference was not statistically significant. Most of patients in the paclitaxel group were censored as they hadn't reached progression yet. Toxicity profile was as expected.

No conflict of interest.

\section{CPC-045 EFFICACY AND SAFETY OF BOCEPREVIR AND TELAPREVIR AT WEEK 12 OF TREATMENT}

doi:10.1136/ejhpharm-2013-000276.502

M Gomez Perez, M Bonilla Porras, B Rodriguez Vargas, FJ Becares Martinez, E Tortajada Esteban, G Toledano Mayoral. Hospital Universitario Fundacion Jimenez Diaz, Hospital Pharmacy, Madrid, Spain

Background Boceprevir and telaprevir are two new drugs approved by the European Medicines Agency for the treatment of hepatitis C genotype 1 . They are used in combination with ribavirin and peginterferon to increase the response to treatment.

Purpose To analyse the evolution of the viral load and the adverse effects of boceprevir and telaprevir, at week 12 of treatment.

Materials and Methods We undertook a prospective observational study from November 2011 to October 2012 of patients who started treatment with boceprevir and telaprevir. Patients were monitored for 12 weeks after initiation of triple therapy. We also analysed the incidence of adverse effects during treatment. The data collected were: age, sex, grade of fibrosis, type of patient, baseline viral load, and viral load at weeks 4, 8 and 12. The data were consulted in the medical records of patients through the IMDHv. 50 programme.

Results A total of 31 patients were followed up, eight treated with boceprevir and 23 with telaprevir. The median of age was 60 years. Regarding the type of patient, 10 were treatment naïve, 5 were relapsers, 7 non-responders, 4 presented side effects in previous treatment and 5 were partial non-responders. The median viral load was 2,682,000 IU/ml. At week 12, undetectable viral load was found in $26(83.8 \%)$ patients ( 6 in the boceprevir group and 20 in the telaprevir group). Five patients $(16.1 \%)$ had to discontinue treatment, four $(12.9 \%)$ had $>1000 \mathrm{IU} / \mathrm{ml}$ at week 12 and one (3\%) due to pancreatitis. Adverse events observed during treatment are shown in the table.

Conclusions The data show an early decrease in the viral load of patients treated with triple therapy, becoming undetectable by week 12 in most cases. The side effects differed from those described in clinical trials, so more studies and post-marketing pharmacovigilance are needed. 


\section{Abstract CPC-045 Table 1}

\begin{tabular}{lll}
\hline & Boceprevir & Telaprevir \\
\hline Thrombocytopenia & 3 & 10 \\
Asthenia & 2 & 14 \\
Anaemia & 2 & 10 \\
Skin Lesions & 1 & 9 \\
Pruritus & & 7 \\
Vitreous Detachment & 1 & 1 \\
Pancytopenia & & 1 \\
Pancreatitis &
\end{tabular}

No conflict of interest.

\section{CPC-046 EPIDEMIOLOGY, SYMPTOMS AND CHEMOTHERAPY OF IMPORTED MALARIA AT MOHAMMED V MILITARY TEACHING HOSPITAL IN RABAT, MOROCCO}

doi:10.1136/ejhpharm-2013-000276.503

'W Enneffah, ${ }^{2} \mathrm{H}$ Naoui, ${ }^{3} \mathrm{~S}$ Makram, ${ }^{4} \mathrm{~J}$ Lamsaouri, 'A Bennana, ${ }^{2} \mathrm{~B}$ Lmimouni. 'Mohammed V Military Teaching Hospital - Faculty of Medicine and Pharmacy, Clinical Pharmacy - Therapeutic Chemistry, Rabat, Morocco; ${ }^{2}$ Mohammed V Military Teaching Hospital - Faculty of Medicine and Pharmacy, Parasitology, Rabat, Morocco' ${ }^{3}$ Mohammed V Military Teaching Hospital - Faculty of Medicine and Pharmacy, Pharmacy - Pharmacology, Rabat, Morocco; ${ }^{4}$ Faculty of Medicine and Pharmacy - Mohammed V University Souissi, Clinical Pharmacy - Therapeutic Chemistry, Rabat, Morocco

Background In Morocco, since the neutralisation of the last outbreak of Plasmodium vivax in 2004, only imported malaria cases have been recorded, the majority from sub-Saharan Africa. At Mohammed V Military Teaching Hospital in Rabat, patients are mostly military, often called to perform missions in malaria endemic areas.

Purpose To report the incidence, origins, symptoms and treatment of malaria at Mohammed V Military Teaching Hospital.

Materials and Methods A prospective study performed from 1 January 2000 to 15 November 2009. All patients who had travelled to a country where malaria is endemic and diagnosed positive for Plasmodium spp in our hospital were included. The data collected concerned the epidemiology, symptoms, diagnosis and treatment of malaria.

Results 145 patients had a thick blood smear positive for malaria parasites. 84\% were Moroccan, the sex ratio Male/Female was 19.71 and the age varied from 6 to 60 years with a median of 34 years. Countries at the origin of the infection were classified in zone 3 in $92 \%$ of cases. All malaria patients were symptomatic at admission, with often one or more of the following symptoms: fever (99\%), chills $(57 \%)$, sweats $(41 \%)$, headaches and various pains $(80 \%)$, vomiting (67\%), nausea (44\%), anaemia (44\%) and thrombopenia (73\%). We distinguished 19 cases of severe malaria and 3 cases of probable evolutive visceral malaria unconfirmed by serology.

Plasmodium falciparum was responsible for most cases, alone in $68 \%$ of cases and in combination with other Plasmodium species in $10 \%$ of cases. A diagnosis was made within three months of returning from the endemic malaria area for $97 \%$ of cases. The drugs most commonly used for treatment were mefloquine (25\%), quinine $(17 \%)$ and the combination of the two $(50 \%)$.

Conclusions This study allowed us to better understand the profile of our malaria patients in order to improve their management in our hospital.

No conflict of interest.

\section{CPC-047 EPILEPSY MANAGEMENT FROM THE CLINICAL PHARMACIST'S POINT OF VIEW AMONG EPILEPSY OUTPATIENTS IN THE EASTERN HUNGARIAN DATABASE}

doi:10.1136/ejhpharm-2013-000276.504

'L Horváth, ${ }^{2} \mathrm{~K}$ Fekete, 'E Csótó, ${ }^{2} \mathrm{~F}$ Fekete. 'University of Debrecen, Central Pharmacy, Debrecen, Hungary; ZUniversity of Debrecen, Department of Neurology, Debrecen, Hungary
Background Epilepsy may need chronic medical treatment throughout life. This is why, besides epileptologists, clinical pharmacists also have an important role in the evaluation of effectiveness, tolerability, side effect, drug interaction, teratogenicity of antiepileptic drugs (AEs).

Purpose To investigate how the cooperation of epileptologists and clinical pharmacists influence compliance and the effect of AEs on the quality of life.

Materials and Methods We analysed 60 parameters of 1845 adult outpatients with epilepsy in the Eastern-Hungarian Database at the Department of Neurology, between 1992-2011. The clinical pharmacist collected and analysed data from 1015 men and 830 women that were related to epilepsy treatment. For statistical analysis the 'STATISTICS for Windows' programme was used.

Results The mean age was 49.3 years. Seventy-seven patients had idiopathic and 1768 symptomatic or cryptogenic epilepsy. During the examination period 1517 patients took antiepileptic treatment: $71 \%$ monotherapy, $21 \%$ dual therapy and only $8 \%$ polytherapy. Thirty-eight percent of the patients were on carbamazepine and $14 \%$ valproate monotherapy. Seventeen percent of the patients were seizure-free on levetiracetam, lamotrigine or oxcarbazepine monotherapy at least for one year. The ratio of side effect was $7.6 \%$. Eighty-eight patients gave birth, 70 of whom took AEs during the organogenesis. No minor or major developmental disorders were observed, although there was one spontaneous miscarriage. At the start of the study a surprisingly high proportion of the patients $(36.2 \%)$ received concomitant treatment affecting the CNS that could also influence the AEs metabolism. After carefully analysing the patient's history and symptoms, we could decrease the use of the co-medication (diazepam, antidepressants, minor and major tranquillisers, alprazolam) to $14.6 \%$ of the patients. The compliance was good in $78.7 \%$ of the patients.

Conclusions The data of Epilepsy Database analysis may give useful information in clinical practise, not only for epileptologists but clinical pharmacists too. Individuall-planned monotherapy decreases the side effects and improves the quality of life in patients with epilepsy.

No conflict of interest.

\section{CPC-048 ESTABLISHING THE ROLE OF THE PHARMACIST IN AN INPATIENT ANTICOAGULATION MANAGEMENT SERVICE IN BELGIUM}

doi:10.1136/ejhpharm-2013-000276.505

'A Vervacke, 'S Lorent, ${ }^{2}$ S Motte. 'Erasme University Hospital, Pharmacy, Bruxelles, Belgium; ${ }^{2}$ Erasme University Hospital, Thrombosis Unit, Bruxelles, Belgium

Background The complexity of the management of vitamin $\mathrm{K}$ antagonist (VKA) treatment has led to the development in many countries of anticoagulant management services (AMS) which provide patient education and good family physician communication in a systematic and coordinated fashion. In Belgium, there is only limited experience in AMS.

Purpose To determine the impact of a pharmacist-provided anticoagulation management programme (AMP) aiming at improving patient education and communication with the family physician.

Materials and Methods This was a prospective cohort study including consecutive inpatients newly initiated on VKA in an urban teaching tertiary care hospital. Patients and general practitioners were interviewed by phone shortly after discharge by using a standardised questionnaire to evaluate the quality of patient education and the quality of discharge reports before (usual care) and after implementation of a pharmacist-provided AMP. The AMP provided structured patient education and a standardised discharge report for family physicians. 